\title{
Lessons Learned in Using Social Media for Disaster Relief - ASU Crisis Response Game
}

\author{
Mohammad-Ali Abbasi†, Shamanth Kumar†, Jose Augusto Andrade Filho $\ddagger$ \\ Huan Liu† \\ $\dagger$ Computer Science and Engineering, Arizona State University \\ $\ddagger$ Department of Computer Science-ICMC, University of Sao Paulo \\ (Ali.Abbasi, Skumar34, Huan.Liu)@asu .edu, Augustoa@icmc.usp.br
}

\begin{abstract}
In disasters such as the earthquake in Haiti and the tsunami in Japan, people used social media to ask for help or report injuries. The popularity, efficiency, and ease of use of social media has led to its pervasive use during the disaster. This creates a pool of timely reports about the disaster, injuries, and help requests. This offers an alternative opportunity for first responders and disaster relief organizations to collect information about the disaster, victims, and their needs. It also presents a challenge for these organizations to aggregate and process the requests from different social media. Given the sheer volume of requests, it is necessary to filter reports and select those of high priority for decision making. Little is known about how the two phases should be smoothly integrated. In this paper we report the use of social media during a simulated crisis and crisis response process, the ASU Crisis Response Game. Its main objective is to creat a training capability to understand how to use social media in crisis. We report lessons learned from this exercise that may benefit first responders and NGOs who use social media to manage relief efforts during the disaster.
\end{abstract}

\section{Introduction}

Social Media is a term ascribed to the current generation of Internet-based social information sharing and social interaction platforms. Some popular examples of social media are Facebook, Twitter, Youtube, and Flickr. Advances in mobile devices, have allowed social media to become available and accessible to anyone who is connected to the Internet. Social media such as Twitter has even let users share microblogging messages via SMS.

In August, 2010, Red Cross published a report ${ }^{1}$ that for the first time surveyed 1,054 respondents from the United States population and reported on the expectations from and usage of social media during disasters. The study discovered that Facebook was the most popular social media website with more than $58 \%$ of users maintaining a Facebook account and it was also the most preferred channel for posting eyewitness information or sending information about

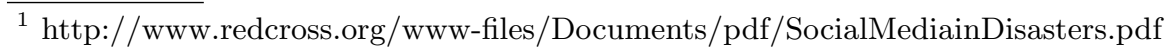


one's safety. The study also discovered that population between 18-35 was more likely to use social media to send and receive information during emergencies. The number of users on social media sites has been constantly increasing. Now Facebook has more than 800 million users and over $75 \%$ of these users are from outside the United States ${ }^{2}$. Twitter, a popular microblogging site has more than 200 million users. Several million messages are posted on these two sites everyday and it is clear that they can play a key role as an information source during disasters. In the light of these facts, the results from the ASU Crisis Response Game show that people do have expectations from social media that must be addressed by agencies responding to a disaster. Moreover, it shows that social media can be a valuable means to reach out to people and offer assistance.

In the past, social media has been used to publish eyewitness accounts after a disaster. Twitter was one of the first sources of eyewitness information during the Mumbai terror attacks in $2008^{3}$. After the earthquake in Japan in early 2011, a Japanese Twitter user reached out to the American Ambassador in Japan, John Roos, who was heading the American rescue operations after the earthquake. With the following tweet: "Kameda hospital in Chiba needs to transfer 80 patients from Kyoritsu hospital in Iwaki city, just outside of $30 \mathrm{~km}(\mathrm{sic})$ range" ${ }^{4}$. Social media can be a valuable source of information to obtain situational awareness during and after a disaster. More recently, the disaster relief agencies have recognized the potential of social media as an information outlet. Hurricane Irene was the first natural disaster where the official agencies used social media to spread information about disaster awareness and preparation ${ }^{5}$.

A recent Congressional research report [6] on social media outlines how social media was used by agencies during disasters. The focus of agencies is now shifting from passively observing on social media to actively communicating with people. Social media has been used previously for disseminating disaster preparation information and at times for community outreach. Obtaining situational awareness through monitoring of information flow is another utility of social media sources as outlined above. The report also suggests several ways in which an agency like FEMA can use social media in disaster recovery efforts.

Existing HA/DR systems that aid agencies need to adapt to the changing focus of the agencies. For this change to occur, we think it is essential to understand how information from a disaster can be effectively harnessed. A suitable way to start this process is by testing the systems in a real environment. However, due to the nature of the domain this is not advisable. Therefore, we need a simulated controlled environment where we can conduct these tests. Hence, we create a disaster simulation game that can be played with real people to simulate a disaster and test the systems for crisis and disaster response.

\footnotetext{
${ }^{2}$ http://www.facebook.com/press/info.php?statistics

${ }^{3}$ http://www.guardian.co.uk/technology/2008/nov/27/mumbai-terror-attackstwitter-flickr

${ }^{4}$ http://www.usatoday.com/tech/news/2011-04-12-1Ajapansocialmedia12_CV_N.htm

${ }^{5}$ http://www.huffingtonpost.com/2011/08/28/hurricane-irene-femaresponse_n_939545.html
} 


\section{Related Work}

Disaster simulation games have been played prior to our game. Exercise 24 was one of the first humanitarian assistance and disaster relief exercise to test the usage of social media and communication tools in response to a simulated disaster. First conducted in September 24, 2010, an earthquake and a tsunami were simulated off the coast of California. People were encouraged to create social media accounts and participate in the testing of social media sources. In the second installment of this event conducted on March 28, 2011 ${ }^{6}$, the USEUCOM and the San Diego State University's Viz Lab, simulated an earthquake in the Adriatic Sea off the coast of Montenegro. Volunteers on site participated using social media and programmed messages were posted at each of the three stages of the disaster. Several volunteers assisted in mapping and geolocating responses which were collected and visualized using a Ushahidi ${ }^{7}$ based crowdmap.

Another disaster simulation, the Great California Shake Out ${ }^{8}$, was conducted in October 20,2011. Nearly 9 million people participated in this exercise where people were encouraged to imagine that an earthquake had hit California and to perform the "Drop, Cover, and Hold On" procedure. Although users were not required to publish any information on social media in this simulation, the size of the population that participated is of interest.

The exercises mentioned above focus on testing the ability to collect information from social media and preparing people for a disaster. In order to effectively use social media for disaster response, we need to be able to collect reports from the various social media sources and act upon the ones that require a response. Trustworthiness of such data has been of some concern lately as social media is a free medium. This issue was investigated in the study performed by Mendoza et.al. [7] on tweets generated during the 2010 Chilean earthquake. The authors discovered that immediately after the earthquake several rumors were posted on Twitter which increased the already existing chaos after the disaster. In a subsequent study [1] they propose a model based on features constructed using the qualities of the user, tweet, topic, and propagation that can be used to predict the credibility of a message. A system to detect rumors and misuses of Twitter has also been built by researchers at the University of Indiana, called Truthy ${ }^{9}$.

Crowdsourcing is one of the approaches that demonstrate the power of social media. Crisis maps are one type of social media based system which are being used more prominently for disaster response during crises. Authors Gao et.al. discuss some existing systems using examples from crisis maps deployed for real disasters [2]. One such platform is Ushahidi which can collect, organize, and visualize SMS reports on a map. Challenges in using this system include a manual verification and preprocessing step necessary to ensure that the received information is accurate and actionable. At $\mathrm{NSCWDD}^{10}$, the Quicknets team has built

\footnotetext{
${ }^{6}$ https://sites.google.com/a/inrelief.org/24/media-report

7 http://www.ushahidi.com/

8 http://www.shakeout.org/

9 http://truthy.edu

10 http://www.navsea.navy.mil/nswc/dahlgren/default.aspx
} 
a plugin for the Ushahidi system which facilitates identification of actionable information by trained volunteers from organizations, such as Humanity Road Inc. It is also capable of replying back to a user via SMS to request additional information or to send an update on the response. Quicknets focuses on SMS but can be potentially used with other social media for disaster relief. In [4], the author outlines some of the problems with existing crisis map platforms and suggests guidelines that will help in the future development of such systems. Recently, Twitter has attracted a lot of attention for its role in various disasters. It represents one popular way to produce timely and instant data. At ASU, we have built a Twitter monitoring and analysis system - TweetTracker [5] that can be easily customized for tracking and retrieving disaster-related information to assist first responders to make critical decision and effective response. These systems concentrate on using the information from social media to respond to a disaster. Such a response has not been tested previously.

\section{ASU Crisis Response Game}

The game is a live-action role-playing exercise in which volunteers take part as either victims or first-responders involved in a disaster. In this game, there is intensive use of Twitter and SMS. In the game victims use social media to ask for help. These requests are collected and processed by a relief system (TweetTracker or QuickNets). Based on these reports, missions will be generated upon which the first-responders can act. Figure 1, shows the architecture of the game.

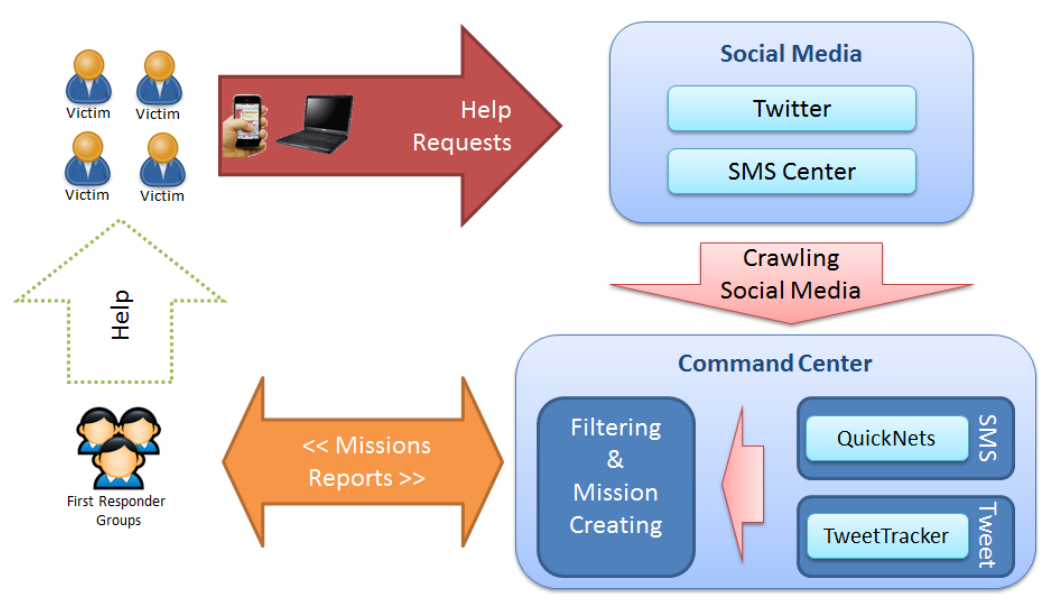

Fig. 1: ASU Crisis Response Game, Architecture

\subsection{Game Components}

There are three main components, Victims, First-responders, and Filtering team. 
Teams Victim (TV) These teams perform the role of victims in a disaster scenario. They use social media (Twitter in this case) or send SMS to ask for help or report injuries. In each message they should include their location, a specific game related hashtag, and their request. They can report any kind of problem, such as fire, rioting, injuries, among others. Individuals or a group of two or more people make a team. Each team should have a device for communication (e.g., smart phone, laptop, or tablet) with ability of connecting to the Internet to send tweets or use their phones to send SMS. At the beginning of the game Victims scatter themselves around the pre-defined game locations. They only can send requests when they are settled in the game locations. Then they should stay till first-responders arrive to resolve their problem. Using social media or sending SMS to a center is the only way that victims can communicate with relief organizations. Teams should activate geo-location related features on their device when they use it to ask for help. This feature helps first-responder teams to locate them easily.

First-Responder Teams (FR) These teams perform the role of NGOs, government agencies, and any other organization able to help. They use a relief coordination system (QuickNets, ACT or TweetTracker in the ASU Game) to find requests for which they can take responsibility. Each team is composed of two or more volunteers. Each team member has one or more capabilities such as Security, Fire and Rescue, Medical, and Wildcard. Teams can perform missions according to their capabilities. In addition some teams can have Wildcard which enables teams to perform all kind of missions. At the beginning of the game, first-responders are at their designated homebase. After Victims send requests for help, FRs select missions based on team members' capabilities and go to the TV's location. Team-Victim members have to stay in place until first-responders arrive and fulfill their request. If the FR team does not have all of the necessary capabilities they should collaborate with other FR teams to accomplish the mission. When a problem has been solved they report back the new situation to the center. When they run out of resource cards to spend, they must go back to their head quarter and get resources renewed.

Filtering Team During a disaster people send many tweets about the disaster. The filtering team is responsible for reviewing the tweets and selecting ones that are related to the mission of first responders. After the selection process they generate missions. These missions includes details about the problem and the location. Each mission can be generated by analyzing one or more tweets. It is possible that they generate more than one mission from a single tweet. This team is using specific software that is able to collect data from social media or receive SMS from victims. Then they use their own system to publish missions that will be used by FR. This team is responsible for generating missions. To do this task they need to perform the following sub-tasks:

- Selection (Deciding whether a message is actionable) 
- Categorization (for the game, what capabilities are necessary to do the task)

- Geolocation (Finding the exact locating of the victims)

These tasks can be done manually or by using software systems. After this step, first-responders will be able to see and select the missions.

\section{Game Exercise and Lessons Learned}

We ran our game during the last week of the August 2011, at ASU main campus. Over 75 volunteer students participated in the real game and played for more than 4 hours. We assigned them to 25 teams of victims, and 8 teams of FirstResponders. In addition around 20 more people participated in the game as the Filtering team and Line-Judges ${ }^{11}$ Victims had the opportunity to scatter into 7 different buildings in the campus as shown in Figure 2. Finally, $17(68 \%)$ Victim teams and $4(50 \%)$ First-Responder teams visited at least 1 out of 7 game location which is labeled on the ASU map. All of the seven buildings were visited by both teams. We collected 212 Short Messages (SMS) and 230 tweets from 13 distinct tweeters. Victims used \#ASUGAME as part of their tweets to make it possible to be found by the First-Responder's twitter crawler software.

\subsection{Social Media Data Collection}

TweetTracker was used to collect tweets from Twitter. In addition, we used ACT and QuickNets to collect SMS. TweetTracker is a tool for collecting and analyzing tweets to obtain situational awareness. ACT (ASU Coordination Tracker) is a tool for crisis event visualization, communication, monitoring, and coordination [3]. QuickNets is a plugin of Ushahidi for NGOs to collect and respond to SMS and email requests that have been validated by domain experts.

\subsection{Lessons Learned}

Many valuable lessons are learned. We summarize the major ones below.

Collecting tweets. Each day people use Twitter to send more than 200 million tweets. Employing a proper method to find related tweets is very important. In this game, we used two different systems to collect tweets. One uses Twitter streaming API to collect and the other uses the jTwitter API to increase coverage. Twitter steaming API does not have limitation but jTwitter has the limitation of 150 or 20,000 requests per hour for regular and white-list users, respectively. In both methods we searched for tweets that mentioned the hashtag \#ASUGAME. The most reliable way to find tweets related to a crisis is by searching for hashtags that people create and use during the crisis.

Creating FR missions. The filtering team was responsible for reviewing all of the tweets and generating missions based on the tweets. Even during the ASUGame in which we only had 220 tweets our filtering team was overloaded. In a

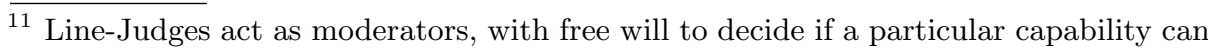
be applied to a particular mission or not. They also help volunteers with any doubts. 


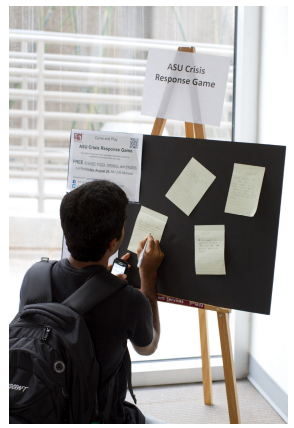

(a)

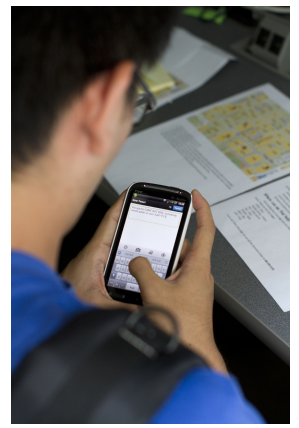

(b)

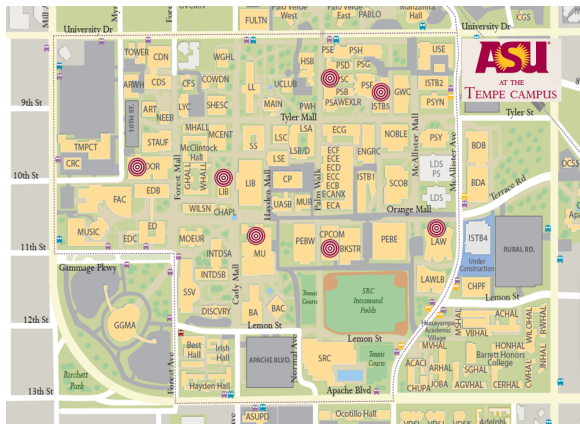

(c)

Fig. 2: (a) and (b) show victims that tweeting and posting a help request during the game. (c) Shows the game map. We used 7 locations (which are highlighted by a circle) in ASU main campus to simulate the real disaster relief.

real crisis, the number of tweets and SMSs would be significantly higher, thus we must use automated systems. Finding related and actionable tweets is the most complicated task in this process. During the disaster a small fraction of tweets are related and can be used to create missions. To find that tiny percent, all of the tweets should be processed. In addition, there are many conversational tweets about the crisis that using the same hashtags and keywords used by victims. Spammers are another problem that generate lots of tweets that should be found and ignored. A practical way to handle this process is using a system that rank tweets according to their importance for a specific crisis. One practical way to help first responders is asking victims to include as much information as they can in their tweets. For example, we asked each team to include location and team identification. This task consumed resources and quickly became a bottleneck. Two solutions for this problem are (1) to develop an intelligent analytic system that can prioritize the tweets or (2) to recruit a sufficient number of experienced people to work for the Filtering team.

GeoLocation People are able to attach their location information to new Tweets through the web and mobile clients. The knowledge of the exact location of victims and areas that require assistance is invaluable during disasters. In the past, we have observed that less than $5 \%$ of users provide location information with their tweets due to privacy concerns and lack of awareness about this feature. In the game, even after being given explicit instruction to add location information to their tweets only few of the tweets had geotag.

NOT REAL THIS IS A GAME! During the gameplay we were concerned that our tweets might be observed by a real agency and they might be mistaken to have been generated in response to a real disaster in ASU. We coordinated with the campus police department and instructed the participants to begin their messages with "NOT REAL THIS IS A GAME!" 
Languages In crises such as Haiti's earthquake people used different languages to tweet. The crawler should be able to search among tweets with popular languages in the area that disaster happens. In the game, we encouraged victims to use languages other than English, and tried to have one non-English speaker in each team if possible. Translation is critical for information gathering and integration and could be time-consuming and error-prone.

\section{Conclusions}

ASU Crisis Response Game simulates a disaster scenario (e.g., earthquakes) and tests how social media based disaster relief systems would work in a crisis simulation on ASU campus. Around 75 students voluntarily played different roles (victims and first responders) in this disaster scenario. In the simulation, victims had sent out their requests using Twitter or SMS. First-responders used different software to collect the requests from victims and manage actions, and responses. The process helped us evaluate how these systems work on real situations. The experience of using social media during a controlled disaster relief process had valuable lessons for our team.

\section{Acknowledgments}

This research was sponsored in part by the Office of Naval Research (ONR) grant: N000141010091. We would also like to thank members of DMML Lab at ASU and our collaborators Catherine Graham from Humanity Road Inc., Mark Bradshaw and his team from NSWCDD, Office of Joint Staff, Dr. Rebecca Goolsby from ONR, and Professor Kathleen Carley and her team from CMU.

\section{References}

1. C. Castillo, M. Mendoza, and B. Poblete. Information credibility on twitter. In Proceedings of the 20th international conference on World wide web, pages 675-684. ACM, 2011.

2. H. Gao, G. Barbier, and R. Goolsby. Harnessing the crowdsourcing power of social media for disaster relief. Intelligent Systems, IEEE, 26(3):10-14, 2011.

3. H. Gao, X. Wang, G. Barbier, and H. Liu. Promoting coordination for disaster relief-from crowdsourcing to coordination. Social Computing, Behavioral-Cultural Modeling and Prediction, pages 197-204, 2011.

4. R. Goolsby. Social media as crisis platform: The future of community maps/crisis maps. ACM Transactions on Intelligent Systems and Technology (TIST), 1(1):7, 2010.

5. S. Kumar, G. Barbier, M. A. Abbasi, and H. Liu. TweetTracker: An Analysis Tool for Humanitarian and Disaster Relief. In Fifth International AAAI Conference on Weblogs and Social Media (ICWSM), 2011.

6. B. R. Lindsay. Social Media and Disasters: Current Uses, Future Options, and Policy Considerations. CRS Report for Congress, 2011.

7. M. Mendoza, B. Poblete, and C. Castillo. Twitter under crisis: Can we trust what we rt? In Proceedings of the First Workshop on Social Media Analytics, pages 71-79. ACM, 2010. 